\title{
COVID-19 Associated Transient Cytotoxic Lesion of the Corpus Callosum: Report of Two Cases and Current Literature Review
}

\author{
๑ Gizem Gursoy, ๑ Berrin Muberra Uzunalioglu, ๑ Cansu Elmas Tunc, ๑ Zulfikar Memis,

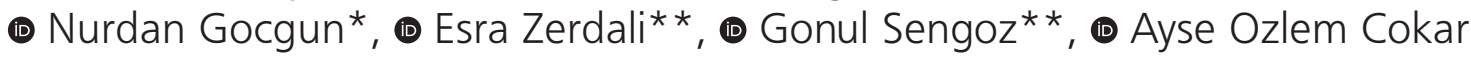 \\ University of Health Sciences Turkey, Haseki Training and Research Hospital, Clinic of Neurology, Istanbul, Turkey \\ *University of Health Sciences Turkey, Haseki Training and Research Hospital, Clinic of Radiology, Istanbul, Turkey \\ **University of Health Sciences Turkey, Haseki Training and Research Hospital, Clinic of Infectious Diseases, Istanbul, Turkey
}

Abstract

Cytotoxic lesion of the corpus callosum (CLOCC) stems from a variety of causes such as malignancies, drug treatments, metabolic disorders, subarachnoid hemorrhage, and infections, and often presents as encephalitis or encephalopathy.

During this pandemic, we saw 2 cases with this lesion; the first one was a 42-year-old male who presented to the emergency department with complaints of headache, fever, and cough. There was a ground-glass opacity in the thorax computed tomography, and diffusion restriction was found in the corpus callosum splenium in the cranial magnetic resonance imaging (MRI) performed for headache that did not resolve with analgesic treatment during hospitalization due to the preliminary diagnosis of Coronavirus diseases-2019 (COVID-19) pneumonia. In the second case, Severe Acute Respiratory syndrome Coronavirus-2 polymerase chain reaction was found to be positive in the examinations performed during his admission to the emergency service due to weakness and presyncope, and diffusion restriction was observed in the corpus callosum splenium like the first case in cranial imaging. The follow-up cranial MRI was normal in both cases, so they were diagnosed with CLOCC.

We aimed to report the present cases with COVID-19 associated CLOCC since they presented only as a headache and a presyncope without any mental deterioration.

Keywords: COVID-19, splenium, corpus callosum, headache, presyncope

\section{Introduction}

Coronavirus disease-2019 (COVID-19) infection has a wide range of symptoms from mild such as coughing and fever to severe such as multiple organ failure, and it is known to have a mortality rate of $2-4 \%$ (1). A study conducted in the province of Wuhan where the outbreak spread from found that the neurological involvement was $36.4 \%$, and that $35.9 \%$ of the patients with neurological involvement had headaches (2).

Cytotoxic lesion of the corpus callosum (CLOCC) is an imaging finding which usually resolves within the first month of the neurological symptom, and develops in a variety of medical conditions including viral infections, epileptic seizures, metabolic disorders, drug toxicity, malignancies, status migrainosus, and cerebrovascular disease (3). While the co-existence of this lesion with COVID-19 was shown, as in our cases, moderate-tosevere encephalopathy has been highlighted in the clinical pictures of most cases; therefore, we aimed to contribute to the literature with our cases having only a headache and a presyncope without any encephalopathy.

\section{Case Report}

\section{Case 1}

A forty-two-year-old male patient without any known chronic diseases presented to the emergency department in July 2020 with headache, fever, coughing, nauseavomiting, and weakness for the past 3 days. In the

Address for Correspondence: Gizem Gursoy, University of Health Sciences Turkey, Haseki Training and Research Hospital, Clinic of Neurology, Istanbul, Turkey Phone: +90 5373024907 E-mail: drgizemgursoy@gmail.com ORCID: orcid.org/0000-0003-4448-5962 Received: 26.02.2021 Accepted: 08.04.2021 
emergency department, body temperature was 38.4 ${ }^{\circ} \mathrm{C}$, blood pressure was $110 / 60 \mathrm{mmHg}$, respiratory rate was $18 /$ minute, and the oxygen saturation was $93 \%$ on room air. When the computed tomography (CT) of the thorax revealed areas of parenchymal consolidation containing air bronchograms in the right upper lobe anterior segment, right lower lobe superior segment, right lower lobe medial basal segment, together with the infiltrations of ground-glass opacity in those areas, the patient was hospitalized with the preliminary diagnosis of COVID-19 pneumonia. Tests revealed high leukocytosis (white blood cell: 13.46 ref. 3.98-10.2 103uL), as well as high levels of markers of systemic inflammation such as C-reaktif protein (CRP) $(235.7 \mathrm{mg} / \mathrm{L}$, ref. $<5)$, ferritin $(919.1 \mathrm{ng} / \mathrm{mL})$, fibrinogen $(603 \mathrm{mg} / \mathrm{dL})$, and D-dimer (10.15 mg/L, ref. <0.55). Nasopharyngeal swab gave a negative result for Severe Acute Respiratory syndrome Coronavirus-2 (SARS-CoV-2) polymerase chain reaction $(P C R)$. It was discovered that the patient about whom we were consulted for a headache had already been having moderate headaches responding to analgesics and lasting for 3 to 4 hours once a week, but the headache at the time of presentation was different from the previous ones and did not ease with analgesics. He described a persistent frontal lobe headache aggravated by coughing and caused by compression he defined as pressure. Having no other accompanying symptoms such as nausea, dizziness, photophobia, and phonophobia, the patient had normal neurological exam results, and his cranial CT gave normal results as well. When the severity of headache did not change following a symptomatic treatment of intravenous $1 \mathrm{~g} / \mathrm{d}$ paracetamol, cranial magnetic resonance imaging (MRI) was used which revealed a nodular lesion with restricted diffusion in the middle part of the splenium of the corpus callosum. The patient whose headache had become less severe as the clinical picture of pneumonia improved and eventually disappeared completely by the time of discharge underwent another cranial imaging 1 month later for the follow-up of the lesion which was then found out to have disappeared (Figure 1).

\section{Case 2}

A fifty-eight-year-old male patient with no known history of any chronic disease presented to the emergency department in July 2020 with weakness, malaise, occasional coughs and presyncope present for the last three days. His body temperature was normal and oxygen saturation was $97 \%$ on room air in the emergency department. Thoracic CT scan revealed images of patchy infectious consolidations in the basal segments of both lungs. The patient was diagnosed with anemia and CRP was found to be $20 \mathrm{mg} / \mathrm{L}$ while procalcitonin was 0.09 $\mathrm{ng} / \mathrm{mL}$ (ref $<0.065 \mathrm{ng} / \mathrm{mL}$ ). Having normal D-dimer and ferritin levels, the patient however tested positive for SARS-CoV-2 by PCR. The brain CT scan aiming to investigate a probable posterior circulation infarct revealed no pathologies other than cavum septum pellicidum while the cervical and cranial CT angiography revealed mild-to-moderate narrowing in segment $\mathrm{V} 4$ of the right vertebral artery. In the diffusion sequence, the MRI scan showed a lesion with restricted diffusion in the splenium of the corpus callosum, and the follow-up cranial MRI scan 2 days later revealed that the restricted diffusion in the splenial region had persisted, and the lesion was still present in the FLAIR sequence. Moreover, the T2FLAIR sequence revealed bilateral multiple hyperintense millimetric regions with subcortical localization and the heme sequence showed several hypointensities consistent with hemorrhage. The follow-up MRI scan 5 days later detected a change in lesion signals consistent with the subacute stage. The EEG test investigating the etiology of presyncope in the patient revealed a basic bioelectrical activity comprising widespread low-amplitude fast waves in both hemispheres, but there was no epileptogenic activity. The follow-up cranial imaging 1.5 months later

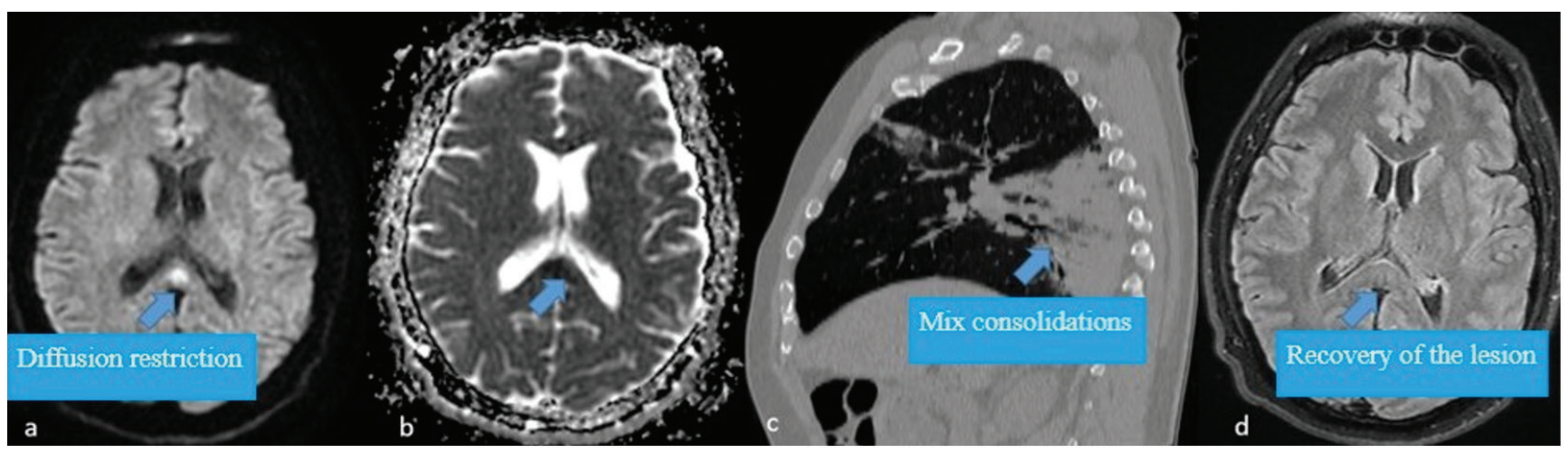

Figure 1. $(a, b)$ Fourty-two years old, the male patient has diffusion restriction in the middle part of splenium (c) Mix consolidations in right upper and lower lobes with bronchograms in the sagittal reconstruction of thorax computed tomography mediastinal window (d) Recovery of the lesion in axial FLAIR sequence without any sequelae or signal 1 month (arrows) 
revealed that the restriction of diffusion had disappeared (Figure 2). Written informed consent was obtained from the patient for publication of these case reports.

\section{Discussion}

CLOCC has been identified to be accompanied by various pathologies including medication-related problems, traumas, subarachnoid hemorrhage, malignancies, metabolic disorders, and most importantly, viral infections by some groups of viruses such as Adenovirus, H1N1 influenza, Epstein-Barr, and Rotavirus (4). While these lesions were previously identified as mild encephalitis/ encephalopathy with a reversible isolated SCC lesion Middle East respiratory syndrome coronavirus, reversible splenial lesions, and reversible splenial lesion syndrome, later the term CLOCC was adopted since not all the cases had mild encephalopathies (they might be non-existent or severe), or they were not restricted only to the splenium (5). Involvement of the corpus callosum manifests itself in one of three models in radiological imaging, i.e. a small circular or oval lesion in the center of the splenium, a lesion centered in the splenium but extending to the adjacent lateral white matter along the callosal fibers, or a lesion found in the posterior region but extending to the anterior corpus callosum (4). This transient lesion which is always found in the center of the splenium in adults has been shown to exist in children as both a small lesion, as in adults, and a lesion extending throughout the corpus callosum and into the parietal white matter, and sometimes even into the frontoparietal white matter (6).

Examination of the pathophysiology of these lesions caused by systemic infection, as in our case, revealed that leukocytes produce proinflammatory cytokines and increase the permeability of the blood-brain barrier and thereby allowing the cytokines and inflammatory cells to enter the central nervous system (CNS). After that the CNS cytokines activate the glial cells (microglia, astrocytes and oligodendrocytes) causing cytotoxic edema through excitotoxic mechanisms $(4,7)$. However, some lesions of the splenium of the corpus callosum secondary to COVID-19 have been shown to be of ischemic nature secondary to hypercoagulability (8). While focal infarction of the corpus callosum is rare since the corpus callosum receives its blood supply from three main arterial systems and there is a pericallosal anastomotic plexus, it is the most common site for the infarction of the splenium. In the acute stage $\mathrm{MRI}$, it presents as diffusion restriction in DWI, high intensity in T2-weighted imaging, and low intensity in T1weighted imaging. It is differentiated from CLOCC since gliosis and atrophy sites develop later and are permanent (9). In a case series of 73 patients with COVID-19 who were scanned retrospectively and underwent cranial MRI, the ratio of diffusion restriction in the splenium of the corpus callosum was determined to be $4.1 \%$ (3 patients), and two of these cases were interpreted to be ischemic. A follow-up MRI of one case on day 25 gave normal results which were interpreted as CLOCC (10).

As the number of cases, worldwide cases increased, retrospective studies on brain imaging have also increased. In a brain imaging study of 7 COVID-19 positive newborns hospitalized due to fever and feeding impairment in Italy, 6 of 7 cases was found a mild reduced diffusion in the genu and, in a lesser extent, in the splenium of the corpus callosum (11). In two different studies, they found that the microhemorrhages were the most common findings, one of our cases had multiple microhemorrhages as supporting these findings $(12,13)$. In a retrospective review by Sawlani et al. (12) microhemorrhages were present in $60 \%$ of patients, with all these patients demonstrating microhaemorrhage in the splenium of the corpus callosum and the most common indication was delirium. In another study includes 16 critically ill patients with COVID-19 who underwent brain MRI because of coma or focal neurologic deficits, diffuse microvascular injury involving the subcortical and deep white matter was detected in $69 \%$ of patients. Microvascular lesions manifested as punctate

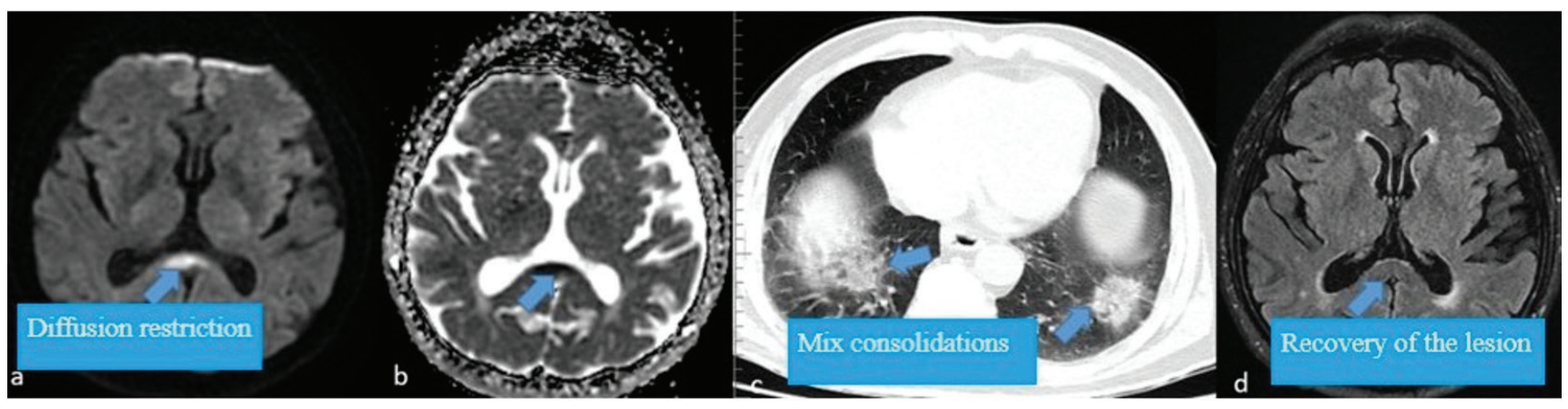

Figure 2. (a, b) Fifty eight years old, male patient with splenial diffusion restriction in DWI (c) Mix consolidations in bilateral posterior segments of lower lobes in axial thorax computed tomography mediastinal window (d) Completely regression of lesion in the axial FLAIR sequence of control cranial magnetic resonance imaging after one and a half month later (arrows) 
and linear hypointense foci on susceptibility-weighted imaging, with a neuroanatomic predilection for the corpus callosum and the subcortical and deep white matter (13).

Literature review revealed a total of 14 cases of CLOCC secondary to COVID-19 5 of whom were pediatric patients and all had mental deterioration of varying degrees. Our cases are the first CLOCC cases reported without any mental deterioration.

\section{Conclusion}

CLOCC may have a variety of causes, the most important one being viral infections. It should be noted that COVID-19 is one of the causes of this lesion and may present without any mental deterioration, and ischemic stroke should be considered in the differential diagnosis.

\section{Acknowledgment}

There is no conflict of interest. We thank Abdullah Karaakın from Beykent University for translating this study from Turkish into English.

\section{Authorship Contributions}

Concept: G.G., N.G., Design: A.D.C., G.S., Data Collection and/or Processing: G.G., Z.M., E.Z., G.S., N.G., B.M.U., C.E.T., Data Collection and/or Processing: G.G., Z.M., C.E.T., B.M.U., Analysis and/or Interpretation: G.G., Literature Research: G.G., Critical Review: A.D.C., G.S., N.G., Writing: G.G., B.M.U.

Conflict of Interest: No conflict of interest was declared by the authors.

Financial Disclosure: The authors declared that this study received no financial support.

\section{References}

1. $W u Y, X u X, C h e n Z$, et al. Nervous system involvement after infection with COVID-19 and other coronaviruses. Brain Behav Immun 2020;87:18-22.

2. Mao $L$, Jin $H$, Wang $M$, et al. Neurologic Manifestations of Hospitalized Patients With Coronavirus Disease 2019 in Wuhan, China. JAMA Neurol 2020;77:683-90.
3. Tetsuka S. Reversible lesion in the splenium of the corpus callosum. Brain Behav 2019;9:e01440.

4. Starkey J, Kobayashi N, Numaguchi Y, Moritani T. Cytotoxic Lesions of the Corpus Callosum That Show Restricted Diffusion: Mechanisms, Causes, and Manifestations. Radiographics 2017;37:562-76.

5. Galnares-Olalde JA, Vázquez-Mézquita AJ, Gómez-Garza G, et al. Cytotoxic Lesions of the Corpus Callosum Caused by Thermogenic Dietary Supplements. AJNR Am J Neuroradiol 2019;40:1304-18.

6. Blaauw J, Meiners LC. The splenium of the corpus callosum: embryology, anatomy, function and imaging with pathophysiological hypothesis. Neuroradiology 2020;62:56385.

7. Starkey J, Moritani T, Kirby P. MRI of CNS fungal infections: review of aspergillosis to histoplasmosis and everything in between. Clin Neuroradiol 2014;24:217-30.

8. Sparr SA, Bieri PL. Infarction of the Splenium of the Corpus Callosum in the Age of COVID-19: A Snapshot in Time. Stroke 2020;51:223-6.

9. Park SE, Choi DS, Shin HS, et al. Splenial Lesions of the Corpus Callosum: Disease Spectrum and MRI Findings. Korean J Radiol 2017;18:710-21.

10. Chougar L, Shor N, Weiss N, et al. Retrospective Observational Study of Brain MRI Findings in Patients with Acute SARSCoV-2 Infection and Neurologic Manifestations. Radiology 2020;297:313-23.

11. Manara O, Barletta A, Pezzetti G, Gerevini S. Brain Imaging Findings in COVID-19 Positive Newborns. Neuroimaging of Covid-19. First Insights based on Clinical Cases 2021;83-6.

12. Sawlani V, Scotton S, Nader K, et al. COVID-19-related intracranial imaging findings: a large single-centre experience. Clin Radiol 2021;76:108-16.

13. Conklin J, Frosch MP, Mukerji SS, et al. Susceptibility-weighted imaging reveals cerebral microvascular injury in severe COVID-19. J Neurol Sci 2021;421:117308. 